\title{
Gitelman Syndrome: A Rare Case of Hypokalaemia and a Novel Mutation
}

\author{
Clara Matos, Fábio Correia, Inês Nunes da Silva, Sofia Carola, Ana Órfão, Maria Ferreira, Teresa Branco \\ Department of Internal Medicine, Hospital Professor Doutor Fernando Fonseca, Amadora, Portugal
}

Doi: 10.12890/2021_002182 - European Journal of Case Reports in Internal Medicine - ○ EFIM 2020

Received: 30/11/2020

Accepted: 01/12/2020

Published: 11/01/2021

How to cite this article: Matos C, Correia F, Silva I, Carola S, Órfão A, Ferreira M, Branco T. Gitelman syndrome: a rare case of hypokalemia and a novel mutation. EJCRIM 2021;8: doi:10.12890/2020_002182.

Conflicts of Interests: The Authors declare that there are no competing interests.

This article is licensed under a Commons Attribution Non-Commercial 4.0 License

\section{ABSTRACT}

Gitelman syndrome (GS) is a hereditary renal tubulopathy caused by mutations in the SLC12A3 gene which encodes the thiazide-sensitive apical sodium-chloride cotransporter. GS is characterized by hypokalaemia, hypomagnesaemia and metabolic alkalosis. Treatment is based on potassium and magnesium replacement ad eternum. We present the case of a young man with palpitations and persistent hypokalaemia, who was diagnosed with GS. Genetic testing revealed 2 mutations in the gene SLC12A3 of combined heterozygosity, both considered pathological. Interestingly, 1 of these mutations was not yet described in the literature or in the reviewed databases. We also discuss the clinical approach and the specificities of managing this rare hereditary renal tubulopathy..

\section{LEARNING POINTS}

- Gitelman syndrome is a rare cause of persistent hypokalaemia.

- A definitive diagnosis is determined by the identification of mutations in the SLC12A3 gene.

- Management consists of chronic potassium and magnesium supplementation aimed at symptom control.

\section{KEYWORDS}

Gitelman syndrome, hypokalaemia, hereditary renal tubulopathy, SLC12A3 mutations

\section{CASE DESCRIPTION}

A previously healthy 22-year-old black man, born in Cape Verde, presented to the Emergency Department complaining of palpitations over the previous 3 days. The physical examination was unremarkable. Blood pressure was 128/61 mmHg and heart rate was 104 bpm. The ECG showed sinus tachycardia with a heart rate of $105 \mathrm{bpm}$, ST depression in D2-D3, aVF and V3-V6, and a prominent U wave from V2-V5. Blood tests revealed a decreased serum potassium level of $2.38 \mathrm{mmol} / \mathrm{l}$, without other electrolyte imbalances and normal serum creatinine. Arterial blood gas analysis showed a metabolic alkalosis: pH 7.48, pCO2 45 mmHg, HCO3 32 mmol/l. There was no history of diarrhoea, laxative or diuretic use or hypertension. The patient was admitted to in-hospital care for further investigation and treatment of severe hypokalaemia. A single voided urine sample showed increased potassium wasting of $64 \mathrm{mmol} / \mathrm{l}$ and a reduced calcium-to-creatinine concentration ratio of 0.019 . Renal ultrasound ruled out nephrolithiasis.

These findings were interpreted as a probable renal tubulopathy. The presentation in a young adult, the absence of nephrolithiasis and a low urinary calcium-to-creatinine translating as hypocalciuria favoured the diagnosis of Gitelman syndrome (GS), as opposed to Bartter syndrome. The patient was treated at first with intravenous potassium chloride until normal potassium serum levels were reached, and then switched to an oral tablet formulation of potassium chloride, taking 4 pills 3 times a day (equivalent to 96 mmol of potassium per day), associated with a mineralocorticoid antagonist, spironolactone, at a dose of $150 \mathrm{mg}$ per day. The patient was discharged and referred to the Internal Medicine outpatient clinic for follow-up. 
Genetic testing later revealed 2 mutations in the SLC12A3 gene of combined heterozygosity, both considered pathological, thus supporting the diagnosis of GS. Interestingly, while the variant c.1928C > T (p.Pro643Leu) had already been described in the literature and in databases [REFs] in association with this syndrome, a new mutation was also identified: c.2085_2095delinsCTGAACAAGA (p.His696*), consisting of the deletion of 11 base pairs and the insertion of a sequence of 10 base pairs, which should have as a consequence the introduction of a premature stop codon in position 696, presumably leading to a non-functional truncated protein. This variant is not yet described in the literature or in the reviewed databases. From the data collected and according to the American College of Medical Genetics and Genomics (ACMG) criteria, this variant is classified as pathological.

Three months following hospital discharge, the patient presented serum potassium levels superior to $3.5 \mathrm{mmol} / \mathrm{l}$ and remained asymptomatic. Due to borderline hypomagnesaemia, he was also started on magnesium pidolate oral solution (130 mg of magnesium per day). He was additionally referred to a Clinical Genetics consultation.

\section{DISCUSSION}

GS, also known as familial hypokalaemia-hypomagnesaemia, is a renal tubulopathy resulting from loss-of-function mutations in the thiazidesensitive apical sodium-chloride cotransporter (NCCT). The prevalence is estimated to be 1:40,000 and mutations in the SLC12A3 gene underlie this tubulopathy, which are inherited in an autosomal recessive fashion ${ }^{[1-3]}$.

GS is characterized by hypokalaemia, hypomagnesaemia, hypocalciuria and metabolic alkalosis ${ }^{[1,2]}$. Decreased reabsorption of sodium chloride in the distal convoluted tubule results in an increased distal tubular flow rate leading to increased potassium secretion through enhanced "maxi" potassium channel activity. Increased distal sodium delivery to the collecting duct induces its reabsorption through ENaC and favours an electronegative tubular gradient that leads to secretion of potassium and hydrogen. Additionally, chronic volume depletion leads to secondary hyperaldosteronism, which exacerbates kaliuresis and metabolic alkalosis, and to increased calcium reabsorption in the proximal tubule resulting in hypocalciuria. Hypomagnesaemia may also occur and is thought to result from down-regulation of TRPM6 channel expression ${ }^{[4]}$.

Clinical features are generally unspecific, including palpitations, muscle weakness, cramps and hypotension. The age of onset is usually around adolescence and early adulthood ${ }^{[1]}$. The differential diagnosis includes other renal tubulopathies, namely Bartter syndrome.

The detection of homozygous or combined heterozygous mutations of the SLC12A3 gene confirms the diagnosis, although 18 to $40 \%$ of clinical GS cases have only 1 mutant allele detected, which may be due to the lack of capacity to detect large genomic rearrangements through conventional techniques such as direct sequencing ${ }^{[3]}$. There are currently 470 registered mutations in the SLC12A3 gene in the Human Gene Mutation Database ${ }^{[5]}$. In the present case, 2 mutations were detected in our patient, including a novel mutation c.2085_2095delinsCTGAACAAGA (p.His696*), which, to the best of our knowledge, has not yet been reported in the literature.

There is no targeted therapy for GS. Life-long potassium and magnesium replacement is the gold standard of treatment, aiming for symptom control. Potassium-sparing diuretics may be used as adjunctive therapy to increase potassium reabsorption in the nephron; however, they may worsen volume depletion, particularly in patients with low dietary sodium intake. ACE inhibitor and NSAID use is controversial and not routinely recommended ${ }^{[1]}$.

This clinical case stands out for the rarity of a syndrome that presents itself as a severe hypokalaemia. High clinical suspicion for this condition is essential to allow early diagnosis, adequate treatment and a better prognosis, preventing adverse consequences, such as arrhythmic complications that can be fatal. Since the treatment is based on potassium and magnesium replacement ad eternum and the need for close monitoring, it is also important to achieve patient compliance.

\section{REFERENCES}

1. Urwin S, Willows J, Sayer J. The challenges of diagnosis and management of Gitelman syndrome. Clin Endocrinol (Oxf) 2020;92:3-10.

2. Knoers NVAM, Levtchenko EN. Gitelman syndrome. Orphanet J Rare Dis 2008;6:1-6.

3. Vargas-Poussou R, Dahan K, Kahila D, Venisse A, Riveira-Munoz E, Debaix H, et al. Spectrum of mutations in Gitelman syndrome. J Am Soc Nephrol 2011;22:693-703.

4. Filippatos TD, Rizos CV, Tzavella E, Elisaf MS. Gitelman syndrome: an analysis of the underlying pathophysiologic mechanisms of acid-base and electrolyte abnormalities. Int Urol Nephrol 2018;50(1):91-96.

5. Human Gene Mutation Database [Internet]. Available from: http://www.hgmd.cf.ac.uk/ac/index.php (accessed 25/11/2020). 